ANNALES

POLONICI MATHEMATICI

XLVIII (1988)

\title{
On a generalization of a theorem of S. Bernstein
}

\author{
by M. Frigon (Montreal) and D. O'ReGAN (Moscow, U.S.A.)
}

\begin{abstract}
In this paper we obtain "weak solutions" via Topological Transversality to nonlinear boundary value problems of the form $y^{\prime \prime}=f\left(t, y, y^{\prime}\right), t \in[0,1]$, with $y$ satisfying appropriatc boundary conditions, where $f:[0,1] \times \boldsymbol{R}^{2} \rightarrow \boldsymbol{R}$ satisfies the Carathéodory Conditions. Our analysis is based on the notions of an essential map and on a priori bounds on solutions.
\end{abstract}

1. Introduction. In this paper we study the existence of solutions to second order boundary value problems of the form

$$
y^{\prime \prime}=f\left(t, y(t), y^{\prime}(t)\right), \quad y \in B, \quad t \in[0,1],
$$

where in fact $f:[0,1] \times \boldsymbol{R}^{2} \rightarrow \boldsymbol{R}$ may be discontinuous. Here of course $\boldsymbol{B}$ denotes suitable boundary conditions. We examine in this paper the case where $f$ satisfies the Carathéordory Conditions, i.e.,

(a) For fixed $(u, v) \in \boldsymbol{R}^{2}, f(a, u, v)$ is Lebesgue measurable on $[0,1]$.

(b) For all $t \in[0,1], f(t, \boldsymbol{a}, \boldsymbol{\square})$ is continuous on $\boldsymbol{R}^{2}$.

For notational purposes let $L^{2}(0,1)$ denote the space of Lebesgue measurable functions $g$ on $(0,1)$ with $\int_{0}^{1}|g(t)|^{2} d t<\infty . L^{2}(0,1)$ with norm $\|g\|_{L^{2}}=\left(\int_{0}^{1}|g(t)|^{2} d t\right)^{1 / 2}$ is a Banach Space.

By a weak solution to (1.1) we mean a function $y \in B$ which together with its derivative $y^{\prime}$ is absolutely continuous on $[0,1]$ with $y^{\prime \prime} \in L^{2}(0,1)$ and $y^{\prime \prime}=f\left(t, y, y^{\prime}\right)$ almost everywhere on $[0,1]$. This paper in fact extends results of Granas, Guenther and Lee [10] which deals with the case where $f$ is continuous. We shall establish, with $f$ satisfying the same physical assumptions as in [10], that (1.1) has bounded weak solutions. Our analysis is based on the Topological Transversality Theorem and known results on Sobolev Spaces.

2. Preliminary notation and results. Let $H^{2}(0,1)$ denote the space of all functions $u$ on the interval $[0,1]$ which are absolutely continuous on $[0,1]$ together with their derivative $u^{\prime}$ and whose derivative $u^{\prime \prime}$ (which exists almost everywhere) is an element of $L^{2}(0,1) . H^{2}(0,1)$ with norm 


$$
\|u\|_{H^{2}}=\|u\|_{L^{2}}+\left\|u^{\prime}\right\|_{L^{2}}+\left\|u^{\prime \prime}\right\|_{L^{2}}
$$

is a Banach Space. Also we let

$$
H_{B}^{2}(0,1)=\left\{u \in H^{2}(0,1): u \in B\right\} .
$$

Finally we state (without proof) some standard theorems which will be used in this paper:

THEOREM 2.1. Let $g$ be a monotone increasing absolutely continuous function on $[a, b]$ with $g(a)=c, g(b)=d$. If $f$ is a nonnegative measurable function on $[c, d]$, then

$$
\int_{c}^{d} f(y) d y=\int_{a}^{b} f(g(x)) g^{\prime}(x) d x
$$

THEOREM 2.2 (Sobolev Imbedding Theorem). $H^{2}(0,1)$ is compactly imbedded into $C^{1}[0,1]$, i.e., the imbedding operator $j: H^{2}(0,1) \rightarrow C^{1}[0,1]$ is continuous and completely continuous.

3. Homogeneous boundary value problems. In this section we examine problems of the form

$$
\begin{aligned}
& y^{\prime \prime}=f\left(t, y, y^{\prime}\right), \quad t \in[0,1], \\
& y \in B,
\end{aligned}
$$

where $f$ is defined in $[0,1] \times \boldsymbol{R}^{2}$. Here $B$ denotes either the boundary conditions

(i) $y(0)=0, y(1)=0$

or

(ii) $-\alpha y(0)+\beta y^{\prime}(0)=0 ; \alpha, \beta>0 ; a y(1)+b y^{\prime}(1)=0 ; a, b>0$.

Now suppose that $f$ satisfies the following hypothesis:

(3.2) $f$ satisfies the Carathéordory Conditions:

(3.3) There is a constant $M \geqslant 0$ such that

$$
\begin{gathered}
y f(t, y, 0)>0 \quad \text { for }|y|>M, \\
|f(t, u, p)| \leqslant A(t, u) p^{2}+B(t, u),
\end{gathered}
$$

where $A(t, u), B(t, u)>0$ are functions bounded on bounded $(t, u)$ sets;

(3.5) $y f(t, y, p)$ is lower semicontinuous at all points

$$
\text { of the form }(t, y, 0) \text {. }
$$

Proposition 3.1. Suppose that $f:[0,1] \times \boldsymbol{R}^{2} \rightarrow \boldsymbol{R}$ satisțies (3.2) and (3.4). 
Then $F: C^{1}[0,1] \rightarrow L^{2}(0,1)$ given by $(F u)(t)=f\left(t, u(t), u^{\prime}(t)\right)$ is defined and continuous.

Proof. Let $\varepsilon>0$ be given and suppose $u_{0} \in C[0,1]$. Consider

$$
\begin{aligned}
G_{\imath m}= & t \in[0,1]:\left\|v-\left(u_{0}(t), u_{0}^{\prime}(t)\right)\right\|<1 / m \\
& \left.\Rightarrow\left|f\left(t, v_{1}, v_{2}\right)-f\left(t, u_{0}(t), u_{0}^{\prime}(t)\right)\right|<\varepsilon / L\right\},
\end{aligned}
$$

where $v=\left(v_{1}, v_{2}\right) \in \boldsymbol{R}^{2},\|v\|=\max \left\{\left|v_{1}\right|,\left|v_{2}\right|\right\}$ and $L$ is a predetermined constant which will be described below. $G_{r m}$ is measurable since $f$ satisfies (3.2). Let $E_{m \varepsilon}=\bigcap_{v \in R^{2}} G_{v m}$. Now $E_{m \varepsilon}$ is measurable and $E_{1 \varepsilon} \subset E_{2 \varepsilon} \subset \ldots$ Also $\bigcup_{m=1}^{\infty} E_{m \varepsilon}$ $=(0,1)$ for if $t_{0} \in(0,1)$, then there exists $m$ such that $\left\|v-\left(u_{0}\left(t_{0}\right), u_{0}^{\prime}\left(t_{0}\right)\right)\right\|$ $<1 / m$, and hence $\left|f\left(t_{0}, v_{1}, v_{2}\right)-f\left(t_{0}, u_{0}\left(t_{0}\right), u_{0}^{\prime}\left(t_{0}\right)\right)\right|<\varepsilon / L$ since $f$ satisfies (3.2). Hence there exists $m_{0} \in N$ such that mes $\left(E_{m_{0^{\varepsilon}}}\right)>1-\varepsilon / L$. Let $A, B$ be constants such that $\left|A\left(t, v_{1}\right)\right| \leqslant A$ and $\left|B\left(t, v_{1}\right)\right| \leqslant B$ for $\left|v_{1}\right| \leqslant 1+\left\|u_{0}\right\|_{1}$, where $\left\|u_{0}\right\|_{1}=\max \left\{\left\|u_{0}\right\|_{\infty},\left\|u_{0}^{\prime}\right\|_{\infty}\right\}$ and $\left\|u_{0}\right\|_{\infty}=\sup _{t \in 0}|u(t)|$. Now for all $\varepsilon>0$ there exists $\eta=\eta(\varepsilon)>0$ such that

$$
\operatorname{mes}(S)<\eta \Rightarrow \int_{S}\left[9 A^{2}\left(u_{0}^{\prime}(t)\right)^{4}+2 B^{2}\right] d t<\frac{1}{3}\left(\frac{1}{2} \varepsilon\right)^{2}
$$

Put

$$
0<\delta<\min \left\{\frac{1}{m_{0}}, \frac{1}{2}\left\{\frac{\varepsilon}{A(6)^{1 / 2}}\right\}^{1 / 2}\right\} \quad \text { and } \quad \max \left\{\frac{\varepsilon}{\eta \eta},(3)^{1 / 2}\right\}<L .
$$

Let $u \in C^{1}[0,1]$ such that $\left\|u-u_{0}\right\|_{1}<\delta$. We will now show that $\left\|F u-F u_{0}\right\|_{I^{2}}<\varepsilon$. If $t \in E_{m_{0}}$, then

$$
\left|f\left(t, u(t), u^{\prime}(t)\right)-f\left(t, u_{0}(t), u_{0}^{\prime}(t)\right)\right|<\varepsilon / L
$$

and so

$$
\int_{E_{m_{0^{E}}}}\left|f\left(t, u(t), u^{\prime}(t)\right)-f\left(t, u_{0}(t), u_{0}^{\prime}(t)\right)\right|^{2} d t<\varepsilon^{2} / L^{2}<\varepsilon^{2} / 3 .
$$

However, mes $\left(E_{m_{0} \varepsilon}^{c}\right)<\varepsilon / L<\varepsilon \eta / \varepsilon=\eta$, and so

$$
\begin{aligned}
& \underset{E_{m_{0} \varepsilon}^{c}}{\int}\left|f\left(t, u(t), u^{\prime}(t)\right)-f\left(t, u_{0}(t), u_{0}^{\prime}(t)\right)\right|^{2} d t \\
& \quad \leqslant 2 \int_{E_{m_{0} \varepsilon}^{c}}\left\{\left|f\left(t, u(t), u^{\prime}(t)\right)\right|^{2}+\left|f\left(t, u_{0}(t), u^{\prime}(t)\right)\right|^{2}\right\} d t \\
& \quad \leqslant 2 \int_{E_{m_{0}}^{c}}\left\{\left|A(t, u(t))\left(u^{\prime}(t)\right)^{2}+B(t, u(t))\right|^{2}+\right. \\
& \left.+\left|A\left(t, u_{0}(t)\right)\left(u_{0}^{\prime}(t)\right)^{2}+B\left(t, u_{0}(t)\right)\right|^{2}\right\} d t
\end{aligned}
$$




$$
\begin{aligned}
& \leqslant 4 \underset{E_{m_{0} \varepsilon}^{c}}{\int}\left(A^{2}\left(u^{\prime}(t)\right)^{4}+B^{2}+A^{2}\left(u_{0}^{\prime}(t)\right)^{4}+B^{2}\right) d t \\
& \leqslant 4 \int_{E_{m_{0} \varepsilon}^{c}}\left\{8 A^{2}\left(\left|u^{\prime}(t)-u_{0}^{\prime}(t)\right|^{4}+\left|u_{0}^{\prime}(t)\right|^{4}\right)+A^{2}\left|u_{0}^{\prime}(t)\right|^{4}+2 B^{2}\right\} d t \\
& \leqslant 4 \int_{E_{m_{0} \varepsilon}^{c}}\left\{9 A^{2}\left|u_{0}^{\prime}(t)\right|^{4}+2 B^{2}+8 A^{2}\left|u_{0}^{\prime}(t)-u^{\prime}(t)\right|^{4}\right\} d t \\
& <4\left\{\frac{1}{3}\left(\frac{1}{2} \varepsilon\right)^{2}+8 A^{2} \delta^{4}\right\}<\frac{1}{3} \varepsilon^{2}+\frac{1}{3} \varepsilon^{2}=\frac{2}{3} \varepsilon^{2} .
\end{aligned}
$$

Hence $\left\|F u-F u_{0}\right\|_{L^{2}}<\varepsilon$, so $F: C^{1}[0,1] \rightarrow L^{2}(0,1)$ is continuous.

The Sobolev Imbedding Theorem together with Proposition 3.1 are now used to extend Theorem 2.1 of [11] for the new class of problems (3.1).

Theorem 3.2 Let, $f:[0,1] \times \boldsymbol{R}^{2} \rightarrow \boldsymbol{R}$ satisfy (3.2), (3.4) and $0 \leqslant \lambda \leqslant 1$. Suppose there is a constant $K$ independent of $\lambda$ such that $\|y\|_{H^{2}} \leqslant K$ for each solution $y(t)$ to

$$
y^{\prime \prime}=\lambda f\left(t, y, y^{\prime}\right), \quad t \in[0,1], \quad y \in B .
$$

Then the boundary value $(3.1)$ has a solution $y$ in $H^{2}(0,1)$.

Proof. Let $\bar{V}=\left\{u \in H_{B}^{2}(0,1):\|u\|_{H^{2}} \leqslant K+1\right\}$ and define $F_{\lambda}: C^{1}[0,1]$ $\rightarrow L^{2}(0,1)$ by $(F v)(t)=\lambda f\left(t, v(t), v^{\prime}(t)\right)$. Now $F_{\lambda}$ is continuous by Proposition 3.1. We have the imbedding $j: H_{B}^{2}(0,1) \rightarrow C^{1}[0,1]$ completely continuous by Theorem 2.2. Finally we define $N: H_{B}^{2}(0,1) \rightarrow L^{2}(0,1)$ by $N y=y^{\prime \prime}$. It is easy to check $N$ is linear, onto and continuous. To show $N$ is one-toone we observe that the boundary conditions (i) or (ii) imply that $y^{\prime}$ vanishes at least once in $[0,1]$. So if $N y=0$ the absolute continuity of $y$ and $y^{\prime}$ with the above observation implies $y=0$. Thus $N^{-1}$ is a bounded linear operator by Theorem 5.10 of [15]. Now $H_{\lambda}=N^{-1} F_{\lambda} j: \bar{V} \rightarrow H_{B}^{2}(0,1)$ defines a homotopy. It is clear that the fixed points of $H_{\lambda}$ are precisely the solutions to (3.1) $)_{\lambda}$. Now $H_{\lambda}$ is fixed point free on $\partial V$. Moreover, the complete continuity of $j$ together with the continuity of $N^{-1}$ and $F_{\lambda}$ imply that the homotopy $H_{\lambda}$ is compact. Now $H_{0}$ is essential so Theorem 1.5 of [12] implies that $H_{1}$ is essential. Thus (3.1) has a solution.

Next sufficient conditions of $f$ are given which imply a priori bounds for solutions to (3.1). Let $y \in H_{B}^{2}(0,1)$ be a solution to (3.1). Suppose $[y(t)]^{2}$ has a maximum at $t_{0} \in(0,1)$. Then from elementary calculus $y^{\prime}\left(t_{0}\right)=0$.

Theorem 3.3. Suppose $f:[0,1] \times \boldsymbol{R}^{2} \rightarrow \boldsymbol{R}$ satisfies (3.2), (3.5) and (3.3). Then any solution $y$ to (3.1) satisfies

$$
|y(t)| \leqslant M, \quad t \in[0,1] \text {. }
$$


Proof. We first show that $|y|$ cannot have a nonzero maximum at 0 or 1. This is true automatically if $y$ satisfies (i). On the other hand suppose $y$ satisfies (ii) and that $|y|$ has a nonzero maximum at 0 . Then $y(0) y^{\prime}(0) \leqslant 0$. However,

$$
y(0) y^{\prime}(0)=\frac{\beta}{\alpha}\{y(0)\}^{2}>0,
$$

a contradiction. A similiar argument works for the case $t=1$. We conclude that $|y|$ can only have a nonzero maximum at $t_{0} \in(0,1)$. Now assume the maximum of $|y|$ is at $t_{0} \in(0,1)$, so $y^{\prime}\left(t_{0}\right)=0$. Suppose $\left|y\left(t_{0}\right)\right|>M$. Then from (3.3), $y\left(t_{0}\right) f\left(t_{0}, y\left(t_{0}\right), 0\right)>0$. The continuity of $y$ and $y^{\prime}$ together with (3.5) implies there exists a neighborhood $N_{t_{0}}$ of $\left(t_{0}, y\left(t_{0}\right), 0\right)$ such that

$$
y(t) f\left(t, y(t), y^{\prime}(t)\right)>0 \quad \text { for }\left(t, y(t), y^{\prime}(t)\right) \in N_{t_{0}} .
$$

On the other hand $y^{\prime}(t)=\int_{t_{0}}^{t} y^{\prime \prime}(s) d s$ and so Fubini's Theorem implies

$$
y(t)=y\left(t_{0}\right)+\int_{t_{0}}^{t}(t-u) y^{\prime \prime}(u) d u
$$

Thus,

$$
y^{2}(t)=y^{2}\left(t_{0}\right)+2 \int_{i_{0}}^{t}(t-u)\left[y(u) f\left(u, y(u), y^{\prime}(u)\right)\right]+\left[y^{\prime}(u)\right]^{2} d u .
$$

Since $|y|$ has a maximum at $t_{0}$, then for $t$ near $t_{0}$

$$
\int_{t_{0}}^{t}(t-u)\left[y(u) f\left(u, y(u), y^{\prime}(u)\right)\right]+\left[y^{\prime}(u)\right]^{2} d u \leqslant 0
$$

which contradicts (*). Thus $\left|y\left(t_{0}\right)\right| \leqslant M$.

We now prove our basic existence theorem for second order boundary value problems.

THEOREM 3.4. Suppose that $f:[0,1] \times \boldsymbol{R}^{2} \rightarrow \boldsymbol{R}$ satisfies (3.2), (3.3), (3.4) and (3.5). Then the boundary value problem (3.1) has at least one solution in $H^{2}(0,1)$.

Proof. To prove existence of a solution in $H^{2}(0,1)$ we apply Theorem 3.2. To establish a priori bounds for $(3.1)_{\lambda}$, let $y(t)$ be a solution to $(3.1)_{\lambda}$. If $\lambda$ $=0$, we have the unique solution $y \equiv 0$. Otherwise, for $0<\lambda \leqslant 1$, $y f(t, y, 0)>0$ for $|y|>M$ implies $\lambda y f(t, y, 0)>0$ for $|y|>M$. Thus Theorem 3.3 implies $|y| \leqslant M$ for any solution $y$ to $(3.1)_{\lambda}$ and for each $\lambda \in[0,-1]$. Hence $\left(\int_{0}^{1}|y(s)|^{2} d s\right)^{1 / 2} \leqslant M$. Finally we obtain a priori bounds on derivatives of $y$. It is easy to observe that boundary conditions (i) or (ii) imply that $y^{\prime}$ vanishes 
at least once on $[0,1]$, so each point $t \in[0,1]$ for which $y^{\prime}(t) \neq 0$ belongs to an interval $[\mu, v]$ such that $y^{\prime}$ maintains a fixed sign on $[\mu, v]$ and $y^{\prime}(\mu)$ and/or $y^{\prime}(v)$ is zero. Assume that $y^{\prime}(\mu)=0$ and $y^{\prime} \geqslant 0$ on $[\mu, v]$. Thus, with $A_{0}, B_{0}$ denoting upper bounds of $A(t, u), B(t, u)$ respectively for $(t, u) \in[0,1] \times[-M, M]$ and since

$$
\left|\hat{\lambda} f\left(t, y, y^{\prime}\right)\right| \leqslant A_{0}\left(y^{\prime}\right)^{2}+B_{0},
$$

we have

$$
\int_{\mu}^{t} \frac{y^{\prime}}{A_{0}} \frac{(u)\left|y^{\prime \prime}(u)\right|}{\left[y^{\prime}(u)\right]^{2}+B_{0}} d u \leqslant 2 M
$$

For $\mu \leqslant u \leqslant t$

$$
\begin{aligned}
{\left[y^{\prime}(u)\right]^{2} } & =\left|\left[y^{\prime}(u)\right]^{2}\right|=2\left|\int_{\mu}^{u} y^{\prime}(s) y^{\prime \prime}(s) d s\right| \\
& \leqslant 2 \int_{\mu}^{u} y^{\prime}(s)\left|y^{\prime \prime}(s)\right| d s,
\end{aligned}
$$

so

$$
A_{0}\left[y^{\prime}(u)\right]^{2}+B_{0} \leqslant 2 A_{0} \int_{\mu}^{u} y^{\prime}(s)\left|y^{\prime \prime}(s)\right| d s+B_{0} .
$$

Thus the previous inequality implies

$$
\int_{\mu}^{1}\left\{\frac{2 A_{0} y^{\prime}(u)\left|y^{\prime \prime}(u)\right|}{2 A_{0} \int_{\mu}^{u} y^{\prime}(s)\left|y^{\prime \prime}(s)\right| d s+B_{0}}\right\} d u \leqslant 4 A_{0} M .
$$

Theorem 2.1 with $g(u)=2 A_{0} \int_{\mu}^{u} y^{\prime}(s)\left|y^{\prime \prime}(s)\right| d s$ yields

$$
\int_{0}^{g(t)} \frac{d u}{u+B_{0}} \leqslant 4 A_{0} M
$$

and so $g(t) \leqslant B_{0}\left(e^{4 A_{0} M}-1\right)$. Moreover, (3.6) yields

$$
\left[y^{\prime}(t)\right]^{2} \leqslant 2 \int_{\mu}^{1} y^{\prime}(s)\left|y^{\prime \prime}(s)\right| d s \leqslant \frac{B_{0}}{A_{0}}\left(e^{4 A_{0} M}-1\right)
$$

and so

$$
\left|y^{\prime}(t)\right| \leqslant\left\{\frac{B_{0}}{A_{0}}\left(e^{4 A_{0} M}-1\right)\right\}^{1 / 2} \equiv M_{1} .
$$

The other cases are treated similarly and the same bound is obtained. Thus 
$\left|y^{\prime}\right| \leqslant M_{1}$ and, in particular, $\left.\underset{0}{1}\left|y^{\prime}(s)\right|^{2} d s\right)^{1 / 2} \leqslant M_{1}$ for each solution $y$ to $(3.1)_{i}$ and for each $\lambda \in[0,1]$. Also (3.3) and the differential equation yields

$$
\begin{aligned}
\left(\int_{0}^{1}\left|y^{\prime \prime}(t)\right|^{2} d t\right)^{1 / 2} & \leqslant\left(\int_{0}^{1}\left[A_{0}\left(y^{\prime}(t)\right)^{2}+B_{0}\right]^{2} d t\right)^{1 / 2} \\
& \leqslant A_{0} M_{1}^{2}+B_{0} \equiv M_{2} .
\end{aligned}
$$

So $\|y\|_{H^{2}} \leqslant K=M_{0}+M_{1}+M_{2}$ and the existence of a solution to (3.1) is established.

Remark. A priori bounds, independent of $\lambda$, for $y^{\prime}$ and $y^{\prime \prime}$ (assuming we have such a bound on solutions) can be obtained as in Theorem 3.4 if instead of the fact that $y^{\prime}$ vanishes at least once on $[0,1]$, we have

$$
\left|y^{\prime}(\mu)\right| \leqslant K
$$

$K \geqslant 0$ a fixed constant independent of $\lambda$, for some $\mu \in[0,1]$. This result will be used in our analysis of the inhomogeneous problem.

4. Inhomogeneous boundary value problems. We have analogue results for the inhomogeneous problem

$$
\begin{aligned}
& y^{\prime \prime}=f\left(t, y, y^{\prime}\right), \quad t \in[0,1], \\
& y \in B
\end{aligned}
$$

where $B$ denotes either the boundary conditions

(iii) $y(0)=r, y(1)=s$ or

(iv) $-\alpha y(0)+\beta y^{\prime}(0)=r ; \alpha, \beta>0 ; a y(1)+b y^{\prime}(1)=s ; a, b>0$.

THEOREM 4.1 Suppose that $f$ satisfies (3.2), (3.3), (3.4) and (3.5). Then the boundary value problem (4.1) has at least one solution in $H^{2}(0,1)$.

Proof. Consider the family of problems:

$$
\begin{aligned}
& y^{\prime \prime}=\lambda f\left(t, y, y^{\prime}\right), \quad 0 \leqslant \lambda \leqslant 1, \\
& y \in B
\end{aligned}
$$

The existence of a solution in $H^{2}(0,1)$ follows from a slight modification of the proof of Theorem 5.1 of [13] once a priori bounds independent of $\lambda$ are established for solutions $y$ to $(4.1)_{\lambda}$. To establish a priori bounds for $(4.1)_{\lambda}$, let $y(t)$ be a solution to $(4.1)_{\lambda}$. Now if $\lambda=0$ we have a unique solution and thus $|y(t)| \leqslant L$ for some constant $L<\infty$. Otherwise for $0<\lambda \leqslant 1, y f(t, y, 0)>0)$ for $|y|>M$ implies $\lambda y f(t, y, 0)>0$ for $|y|>M$. If $y$ satisfies (iii) it follow: immediately from Theorem 3.3 that 


$$
|y| \leqslant M_{0}=\max \{M,|r|,|s|\} .
$$

On the other hand, if $y$ satisfies (iv) we have

$$
|y| \leqslant M_{1}=\max \{M,|r / \alpha|,|s / a|\} .
$$

To see this suppose that $|y(t)|$ assumes its maximum at $t=0$. Then $y(0) y^{\prime}(0) \leqslant 0$. So

$$
0 \geqslant y(0) \beta y^{\prime}(0)=\alpha(y(0))^{2}\left\{\frac{r}{\alpha y(0)}+1\right\}
$$

and consequently $|y(0)| \leqslant|r / \alpha|$. Likewise $|y(1)| \leqslant|s / a|$ if $|y|$ achieves its maximum at $t=1$. Thus $|y| \leqslant M_{2}=\max \left\{M_{0}, M_{1}, L\right\}$ for any solution $y$ to (4.1) ${ }_{\lambda}$. A priori bounds independent of $\lambda$, for $y^{\prime}$ and $y^{\prime \prime}$ follow from the remark after Theorem 3.4 since it is easy to observe that

$$
\left|y^{\prime}(\mu)\right| \leqslant K
$$

$K \geqslant 0$ a fixed constant independent of $\lambda$, for some point $\mu \in[0,1]$. Thus existence of a solution to (4.1) follows from Theorem 5.1 of [13].

Theorem 4.2 (Granas, Guenther and Lee). Suppose $f:[0,1] \times \boldsymbol{R}^{2} \rightarrow \boldsymbol{R}$ is continuous and satisfies (3.3) and (3.4). Then the solution $u$ of (4.1) is a classical solution.

Proof. Let $u$ be a solution (guaranteed by Theorem 4.1) of (4.1), i.e., $u^{\prime \prime}(t)=f\left(t, u(t), u^{\prime}(t)\right)$ almost everywhere for $t \in[0,1]$. Now with $g(t)$ $=f\left(t, u(t), u^{\prime}(t)\right) \in C[0,1]$ and $u^{\prime \prime}(t)=g(t)$ almost everywhere for $t \in[0,1]$ we have $u \in C^{2}[0,1]$ by the uniqueness of generalized derivatives.

Example (Heat Conduction). Suppose $V$ is an isotropic heat conducting medium with $S$ denoting the surface and $\vec{n}$ the outer normal. We define $u$ $=u(x, t)$ to be the temperature at location $x \in V$ and $t>0$. Also $c=c(x, u)$ denotes the specific heat, $p=p(x, u)$ the density and $k=k(x, u)$ the thermal conductivity. Now the Divergence Theorem and Fouriei's Law together with conservation of energy yields the heat equation

$$
\frac{\partial}{\partial t}(c p u)=\operatorname{div}(k \vec{\nabla} u)+h ; \quad x \in V, t>0,
$$

where $h=h(x, u)$ represents the rate of heat generation by internal sources.

We now set up boundary conditions which describe the heat transfer across $S$. Suppose the surroundings of $V$ are kept at a time independent temperature and that heat radiates into the surroundings (according to Newton's Law of Cooling) at a rate proportional to the temperature difference between $S$ and its surrounding environment. The energy balance of heat flow across $S$ together with Fourier's Law yields 


$$
z(x, u) u(x, t)+\sigma(x, u) \frac{\partial u(x, t)}{\partial n}=g(x) ; \quad x \in S, t>0
$$

where $z \geqslant 0, \sigma \geqslant 0$ and $\sigma+z>0$.

We wish to find a steady state solution (temperature distribution) $y=y(x)$. It will satisfy

$$
\begin{gathered}
\Delta y=-\frac{1}{k}\left\{\vec{\nabla}_{k} \cdot \vec{\nabla}_{y+h\}, \quad x \in V,}\right. \\
z(x, y) y(x)+\sigma(x, y) \frac{\partial u(x)}{\partial n}=g(x), \quad x \in S .
\end{gathered}
$$

Now if $V$ is a rod of unit length and insulated laternal surfaces then the steady state problem is

$$
\begin{aligned}
y^{\prime \prime}= & -\frac{1}{k(x, y)}\left[k_{x}(x, y) y^{\prime}+k_{y}(x, y)\left(y^{\prime}\right)^{2}+h(x, y)\right], \\
& z(0, y(0)) y(0)-\sigma(0, y(0)) y^{\prime}(0)=g(0), \\
& z(1, y(1)) y(1)+\sigma(1, y(1)) y^{\prime}(1)=g(1) .
\end{aligned}
$$

We will assume the case where $z, \sigma$ are independent of temperature and set $\alpha=z(0)>0, \beta=\sigma(0)>0, a=z(1)>0, b=\sigma(1)>0, r=g(0)$ and $s=g(1)$.

So our problem reduces to

$$
\begin{gathered}
y^{\prime \prime}=-\frac{1}{k(x, y)}\left\{k_{x} y^{\prime}+k_{y}\left(y^{\prime}\right)^{2}+h(x, y)\right\} \equiv f\left(x, y, y^{\prime}\right), \\
\alpha y(0)-\beta y^{\prime}(0)=r ; \quad \alpha, \beta>0, \\
a y(1)+b y^{\prime}(1)=s ; \quad a, b>0 .
\end{gathered}
$$

Now we make the following assumptions on $h$ and $k$ :

(4.3) $k_{x}(x, y), k_{y}(x, y)$ are continuous for $(x, y) \in[0,1] \times \boldsymbol{R}$. Also suppose for $(x, y) \in[0,1] \times \boldsymbol{R}, k(x, y)$ is continuous and $k(x, y) \geqslant m>0$, where $m$ is a constant.

(4.4) Suppose $h(x, y)$ is bounded for bounded $(x, y)$ sets. Suppose also $h$ satisfies the Carathéordory Conditions.

$$
y h(x, y)<0 \text { for large }|y| .
$$

$$
\frac{y(x)}{k(x, y)} h(x, y) \text { is lower semicontinuous on }[0,1] \times \mathbf{R} \text {. }
$$

The assumption (4.5) that $y h(x, y)<0$ for large $|y|$ means that the internal heat generation $h(x, y)$ opposes large temperature extremes, i.e., if $y>0$ and $|y|$ large, then $h(x, y)<0$ so heat is removed from the rod by internal sources and the temperature tends to drop. 
Now assumptions (4.3)-(4.6) together with Theorem 4.1 implies that (4.2) has at least one solution in $H^{2}(0,1)$.

Acknowledgements. We would like to thank Professor Andrzej Granas and John $\mathbf{W}$. Lee for their invaluable assistance during the research and writing of this paper.

\section{References}

[1] H. Amann and M. G. Crandall, On some existence theorems for semilinear elliptic equations, Indiana Univ. Math. J. 27 (1978), 779-790.

[2] J. M. Bony, Principe du Maximum dans les espaces de Soboler, C. R. Acad. Sci. Paris, Série A, 265 (1967), 333-336.

[3] H. Brezis, Analyse Fonctionnelle, Théorie et Applications, Masson, Paris 1983.

[4] K. C. Chang, The obstacle problem and partial differential equations with discontinuous nonlincarities, Comm. Pure Appl. Math. 33 (1980), 117-146.

[5] J. Dugundji and A. Granas, Fixed Point Theory, Vol. 1, Monografie Matematyczne, PWN, Warszawa 1982.

[6] S. Fučik, Soluability of Nonlinear Equations and Boundary Value Problems, D. Reidel Pub. Co., Dordrecht-Boston-London 1980.

[7] - and A. Kufner, Nonlinear Differential Equations, Elsevier Scientific Pub. Co., Amsterdam-Oxford-New York 1980.

[8] D. Gilbarg and N. S. Trudinger, Elliptic Partial Differential Equations of Second Order. Springer-Verlag, Berlin-Heidelberg 1977.

[9] A. Granas, Sur la méthode de continuité de Poincaré, C. R. Acad. Sci. Paris 282 (1976), 983-985.

[10] -, R. B. Guenther and J. W. Lee, On a Theorem of S. Bernstein, Pacific J. Math. 74 (1978), 67-82.

[11] -, -, -, Nonlineur Boundary Value Problems for Classes of Ordinary Differential Equations, Rocky Mountain J. of Math. 10 (1980), 35-58.

[12] -. - . -, Topological Transversality I (Some Nonlinear Diffusion Problems), Pacific J. Math. 89 (1980), 53-67.

[13] R. B. Guenther, Problèmes aux limites non linéaires pour ceraines classes déquations differentielles ordinaires, Les Presses de L'Université de Montreal, 1985.

[14] M. A. Kransnosel'skii, Topological Methods in the Theory of Nonlinear Integral Equations, Macmillan Company, New York 1964.

[15] W. Rudin. Real and Complex Analysis, McGraw Hill, New York 1966.

[16] M. M. Vainberg, Variational Methods for the Study of Nonlinear Operators, Holden-Day Inc.. San Francisco-London-Amsterdam 1964.

DEPARTMENT OF MATHEMATICS, UNIVERSITY OF MONTREAL MONTREAL, CANADA

and

DEPARTMENT OF MATHEMATICS AND APPLIFD STATISTICS

UNIVERSITY OF IDAHO, MOSCOW, IDAHO 